March 2019 Volume 8 Issue 1

(c) All rights are reserved by John AL Maxwell.

\section{Synchronous Diaphragmatic Flutter in a Lactating Mare}

Keywords: Lactating mare; Colic; Emergency surgery; Euthanasia SDF; Calcium supplementation; Endurance trial; Metabolic disorder Wean

\section{Introduction}

Synchronous Diaphragmatic Flutter (SDF) in the horse was documented initially in the $19^{\text {th }}$ century and referred to as "Thumps".

It is rarely reported and, in more recent times, it has been seen mainly in horses participating in endurance trials [1].

The condition is considered to be a metabolic disorder of low calcium serum levels induced by a variety of factors [2-6].

This report documents the findings of a case in a lactating mare that suddenly developing symptoms of SDF.

\section{Case Report}

A 4-year-old Cob mare bred on a farm at Denmark, in the south of Western Australia, developed signs the owners considered indicated colic.

The mare had foaled four months previously and because the owners wanted to avoid the development of laminitis, they moved the mare and foal from a lush pasture into a less exuberant one.

They then decided to stable both mare and foal, to allow them to begin handling the foal.

Early on the morning following the stabling, the mare was found to be recumbent and not wanting to rise.

The owners considered that the mare had developed colic and contacted their veterinarian, who, after examining the mare, diagnosed large colon torsion.

At approximately 8:00 am, Flunixin (Flunixin meglumine) Dormosedan (Detomidine) and Torbugesic (Butorphanol tartrate) were administered by the attending veterinarian, who advised the owners to either seek surgical treatment or consider euthanasia.

The client did not want to euthanise the mare and, agreed to the option of emergency surgery. However, they decided not to transport the mare on a 5-hour journey to Murdoch University in Perth, but instead to travel 2-hours to Katanning Regional Veterinary Hospital and seek surgical intervention there.

A further dose of Torbugesic was administered by the veterinarian at noon, immediately prior to the mare being transported to Katanning.

The mare arrived at Katanning after 2:00 pm in the afternoon, some 8-hours after initial signs of distress were observed.

The mare presented subdued, lethargic and with a lowered head. There was little clinical evidence of sweating and the mare was assessed as not being markedly dehydrated. The mare walked into the hospital and was steady on its feet, but exhibited breathing difficulties.
Journal of

Veterinary Science \& Medicine

\author{
John AL Maxwell* \\ Katanning Regional Veterinary Hospital, Australia \\ *Address for correspondence: \\ John AL Maxwell, Katanning Regional Veterinary Hospital, PO Box \\ 273, Katanning. Western Australia, Australia; E-mail: jmaxwell@ka- \\ tanningvet.com.au \\ Submission: 12-February, 2020 \\ Accepted: 27-March, 2020 \\ Published: 31-March, 2020 \\ Copyright: () 2020 FJohn AL Maxwell. This is an open access article \\ distributed under the Creative Commons Attribution License, which \\ permits unrestricted use, distribution, and reproduction in any medium, \\ provided the original work is properly cited.
}

On physical examination, the mare was observed to have very rapid abdominal respiration and on auscultation of the chest, was found to have synchronous heart and respiratory rates of nearly 60 per minute, causing the mare considerable respiratory distress and an exaggerated respiratory sound.

Temperature was $38.4 \mathrm{C}$ and on rectal examination faeces was recovered, the large colon was not markedly distended and the reproductive tract was readily palpated.

Because of these signs, I advised the owners that I considered this a case of "Thumps" and admitted the horse for treatment by calcium supplementation.

The prognosis given was guarded, because this condition had been present for some time and, the horse was exhausted and becoming ataxic.

My previous experience of this condition, although limited, was confined to endurance trails. However, I reasoned that the recent history of parturition, changes in feed and stabling may have precipitated a form of metabolic derangement (hypocalcaemia) that induced the condition.

During the evening the mare became recumbent. However, by the following morning she had recovered normality.

She was standing, moving in a completely co-ordinated fashion, exhibiting normal cardinal signs, bright, alert, eating, drinking and passing faeces.

The mare continued throughout the day as if completely recovered and was discharged into the care of the owners on the following morning.

At discharge, I advised the owners to begin the weaning process, keeping the mare and foal together, but not allowing the foal access to the mother's udder at all times

\section{Discussion}

In my experience, the symptom of SDF is very characteristic and unmistakable. So, here I was presented with a case, not at an endurance event, but in a lactating mare.

Although the circumstances were new to me, the symptom was 
characteristic and I had no hesitation in diagnosing SDF and initiating therapy, although the prognosis was not considered favorable.

In this, I underestimated the recuperative capacity of the mare, for overnight she returned to normality, exhibiting normal cardinal signs as well as being bright, alert, eating and drinking. In fact, in my opinion, the mare appeared anxious to be returned to her foal.

I chose to keep the mare for one extra day to monitor the progress and determine if further medication was necessary.

The literature tends to concentrate on the occurrence of this condition where excess stress is imposed to the horse, as is case in endurance even ting.

In addition to this, there is also another body of material relating to metabolic disturbances which have been termed Hypocalcemia, Milk Fever and Eclampsia. This case is clearly within this latter grouping [1,2,4-6].

In summary, I was presented with a case, originally thought to be a surgical emergency.
On presentation, I diagnosed SDF and applied treatment only for that condition.

I gave no further colic medication. The mare responded favourable to this regime and was returned to the owners recovered.

However, this incident needs documenting, so that other animals, in similar situations, are not sent to high-risk surgery or euthanised.

\section{References}

1. Mansmann RA, Carlson GP, White NA $2^{\text {nd }}$, Milne DW (1974) Synchronous diaphragmatic flutter in horses. J Am Vet Med Assoc 165: 265-270.

2. Ambierg $J(1980)$ Hypocalcemia in the horse. A case report. Nord Vet Med 32: $207-211$

3. Dwyer RM, Thompson LS (1985) "The practical diagnosis and treatment of metabolic conditions in endurance horses". lowa State University Veterinarian 40.

4. Spier SJ (2019) Hypocalcemic tetany in Horses. (Transport tetany, Lactation tetany, Eclampsia) MSD Manual Veterinary Manual.

5. Anon. Hypocalcemic Disorders in Horses - Symptoms, Causes, Diagnosis, Treatment, Recovery, Management, Cost.

6. Taylor P (1996) Heat Stroke, Exhaustion and Synchronous Diaphragmatic Flutter (SDF). In: Dyson S. (Eds) A Guide to the Management of Emergencies at Equine Competitions. British Equine Veterinary Association pp: 112. 